\title{
Fluxos mínimos de veículos para implantação de faixas adicionais em aclives de rodovias de pista simples
}

\author{
Ricardo Almeida de Melo; josé Reynaldo Setti ${ }^{2}$
}

\begin{abstract}
Resumo: Este trabalho trata de uma adaptação do critério de fluxos mínimos de veículos, proposto pela AASHTO, para avaliar a necessidade de implantação de faixas adicionais em aclives de rodovias brasileiras. Os fluxos mínimos de veículos foram obtidos por análise econômica com uso de relação benefício/custo. Os benefícios considerados foram a redução dos custos operacionais dos veículos e dos tempos de viagens, quando implantada a faixa adicional. Os benefícios foram calculados do ganho de velocidade média, redução do tempo de viagem e da porcentagem de veículos em espera nos pelotões; essas informações foram extraídas de mais de 20.000 simulações efetuadas com o simulador TRARR. O custo do investimento foi composto por duas parcelas: custo de construção e custo de manutenção da faixa adicional. Os fluxos mínimos obtidos foram aqueles em que a relação benefício/custo era igual à unidade. O critério mencionado complementa uma proposta de adaptação das diretrizes da AASHTO, para análise da necessidade de faixas adicionais de subida em rodovias de pista simples brasileiras que, se adotadas, irão possibilitar a melhoria do nível de serviço e da segurança do trânsito.
\end{abstract}

\begin{abstract}
This paper reports an adaptation for Brazilian conditions of the upgrade traffic flow rate criterion used by AASHTO in the Green Book to justify the need for a climbing lane. In the research reported in this paper, minimum flows that justify building of climbing lane were obtained through benefit/cost analyses. Costs were assumed to be the sum of construction and maintenance costs for the climbing lane. Benefits included the reduction of operational cost of vehicles and travel time after the construction of the climbing lane. The benefits were estimated from increasing in average upgrade speed and reductions of travel time and platoons in traffic. Data for the economic analyses were obtained of more than 20.000 simulations made using the TRARR simulator. The minimum flow that justifies the need for a climbing lane was assumed to be that for which the benefit/cost ratio equals one. The proposed criterion complements an adaptation of the AASHTO guidelines for climbing lanes to Brazil which, if applied, will increase the quality of service and safety on two-lane highways.
\end{abstract}

\section{INTRODUÇÃO}

As faixas adicionais consistem em obras que podem melhorar a operação de veículos e aumentar a segurança das viagens em trechos sinuosos, ondulados e montanhosos de rodovias de pista simples. Com a implantação desse tipo de melhoria, as manobras de ultrapassagens aumentam de forma significativa, o que resulta em melhoria do nível de serviço da rodovia, devido à redução de veículos que viajam em pelotões, aumento de velocidade e redução do tempo de viagem, além da redução de índices de acidentes, aspecto de grande importância em projetos de rodovias. Para se ter uma idéia, de um registro de três anos de acidentes com transporte de produtos perigosos efetuado em rodovias do estado de São Paulo, apenas 12\% ocorreu em aclives dotados de faixa adicional, enquanto que $85 \%$ ocorreram em trechos sem esse tipo de melhoria (Ferreira, 2003).

Em aclives, os problemas relacionados à operação de veículos são causados pela presença de caminhões

\footnotetext{
${ }^{1}$ Ricardo de Almeida Melo, Universidade Federal da Paraíba, Departamento de Engenharia Civil. João Pessoa, PB, Brasil. (email: ricardo@ct.ufpb.br).

${ }^{2}$ José Reynaldo Setti, Universidade de São Paulo, Escola de Engenharia de São Carlos, Departamento de Transportes. São Carlos, SP, Brasil. (e-mail: jrasetti@usp.br).
}

Manuscrito recebido em 26/6/2007 e aprovado para publicação em 19/9/2007. Este artigo é parte de TRANSPORTES, volume XV, número 1, junho de 2007. ISSN: 1415-7713. carregados que, devido ao baixo desempenho, reduzem a velocidade e provocam a formação de pelotões em rampas ascendentes longas e/ou íngremes. Nessas condições, as oportunidades de ultrapassagens para os veículos mais rápidos são reduzidas e, como conseqüência, os custos operacionais dos veículos e os tempos de viagem aumentam, bem como cresce a possibilidade de ocorrência de acidentes devido às tentativas forçadas de ultrapassagem, ou decorrentes das diferenças de velocidades entre os veículos mais rápidos (automóveis e ônibus) e veículos lentos (caminhões carregados).

A implantação de faixa adicional consiste em uma das soluções para tornar as viagens mais econômicas, seguras e confortáveis. Segundo o Departamento Nacional de Estradas de Rodagem (DNER, 1999), a faixa adicional é uma faixa extra implantada ao lado direito da faixa de rolamento, no sentido ascendente, destinada ao tráfego de veículos lentos. Além disso, as faixas adicionais são obras de melhoria de baixo custo e menor impacto ambiental, se comparadas às obras de duplicação de rodovias.

No Brasil, cerca de $90 \%$ das rodovias pavimentadas é de pista simples, o que representa uma extensão total de aproximadamente 160 mil quilômetros (CNT, 2006). Além do mais, em boa parte dessas rodovias, o crescimento do volume diário médio não será em magnitude suficiente para justificar sua duplicação. Ainda, segundo as estatísticas do Departamento Na- 
cional de Trânsito, em 2005 morreram entre 25.000 e 35.000 pessoas em acidentes de trânsito no Brasil (DENATRAN, 2007). Assim, diante dessas circunstâncias, o potencial de implantação de faixas adicionais em aclives é grande, pois em mais de $80.000 \mathrm{~km}$ de rodovias brasileiras, apenas $20 \%$ trechos de rodovias localizados em terreno ondulado ou montanhoso são dotadas de faixas adicionais, segundo pesquisa realizada pela Confederação Nacional do Transporte (CNT, 2006).

Com o intuito de contribuir com diretrizes para implantação de obras de melhoria em rodovias de pista simples brasileiras, este trabalho tem como objetivo mostrar critério de fluxos mínimos de veículos e porcentagens de caminhões que justificam economicamente a construção de faixas adicionais em aclives.

A definição do critério de fluxos mínimos de veículos consiste em aspecto importante, pois se ocorrer situações em que os caminhões carregados reduzem a velocidade no aclive e a quantidade de veículos rápidos em pelotões é pequena, o investimento na obra pode não ser economicamente viável, porque os benefícios gerados aos usuários são inferiores aos custos de investimento para implantação da faixa adicional.

\section{DIRETRIZES PARA IMPLANTAÇÃO DE FAIXA ADICIONAL EM ACLIVES}

De acordo com a literatura consultada, as diretrizes para implantação de faixas adicionais apresentam similaridades ao considerar aspectos sobre fluxos mínimos de veículos e análise econômica do investimento, além de critérios sobre redução máxima admissível para velocidade de caminhões carregados, avaliação de nível de serviço e aspectos sobre aumento de segurança nas viagens. Neste trabalho foram abordadas as diretrizes da American Association of State Highway and Transportation Officials (AASHTO, 2001) em sua plenitude e em aspectos sobre fluxos mínimos de veículos de critérios existentes em países como Austrália, Canadá, México e Brasil (DMR, 2002; ATU, 1995; MTO, 1990; Mendonza e Mayoral, 1994; DNER, 1999; Melo, 2002).

As propostas da AASHTO (2001), Ministry of Transportation Ontário (MTO, 1990) e Mendonza e Mayoral (1994) são mais restritas, pois estabelecem fluxos mínimos de veículos e porcentagens de caminhões, sem que haja correlação com a geometria do aclive analisado. Com relação às orientações de Queensland Department of Main Roads (DMR, 2002) e Alberta Infrastructure, Alberta Transportation and Utilities (ATU, 1995), os aspectos sobre tráfego são mais abrangentes, visto que os fluxos mínimos estão correlacionados com uma maior magnitude de porcentagens de caminhões, condições de operação do trecho que antecede o aclive, comprimento e declividade da rampa. Mais detalhes sobre essas diretrizes estão descritas nos itens que se seguem.

\subsection{Critérios da AASHTO}

As diretrizes da AASHTO (2001) estão entre as mais difundidas e servem de referência para manuais de projeto de faixas adicionais existentes em diferentes países. Conforme a AASHTO (2001), os critérios que devem ser analisados para justificar uma faixa adicional, considerando aspectos econômicos, são:

1. Taxa de fluxo de veículos no aclive é maior que 200 veíc/h;

2. Taxa de fluxo de caminhões no aclive é maior que 20 veíc/h;

3. Uma das condições é verificada:

a. A redução de velocidade para o veículo de projeto (caminhão carregado com relação massa/potência de $120 \mathrm{Kg} / \mathrm{KW}$ ) é igual ou maior que $15 \mathrm{~km} / \mathrm{h}$;

b. O nível de serviço estimado para o aclive é "E" ou "F";

c. A diferença de dois ou mais níveis de serviços entre o trecho de aproximação e o aclive.

Além disso, caso seja avaliada a necessidade de prover mais segurança durante as viagens no aclive analisado, os índices de acidentes são suficientes para justificar a implantação de uma faixa adicional, sem a necessidade de verificação dos critérios mencionados.

\subsection{Critérios de fluxos mínimos de veículos}

O Queensland Department of Main Roads, na Austrália, define volumes diários médios como um dos critérios de implantação de faixas adicionais em aclives (DMR, 2002). Os valores mínimos de fluxos de veículos são baseados em extensão de zonas de ultrapassagens permitidas e porcentagem de veículos lentos, conforme mostra a Tabela 1.

Em Alberta, Canadá, as diretrizes de implantação de faixas adicionais são baseadas em volumes diários médios em função da porcentagem de ultrapassagem permitida, porcentagem de veículos pesados (automóveis com reboques, ônibus e caminhões) na corrente de tráfego, declividade e comprimento da rampa (ATU, 1995; p. B-64-B-67). Este critério, por sua vez, está baseado em três condições:

1) O volume diário médio de veículos pesados deve ser maior ou igual a 150 veíc/dia;

2) Na hora de pico, o nível de serviço calculado deve ser inferior ao nível "A";

3) Os volumes diários médios devem ocorrer num período menor que 10 anos (metade do horizonte de projeto: 20 anos); e,

4) Os benefícios estimados (custos dos usuários, redução de tempo de viagem e de acidentes) devem proporcionar uma taxa interna de retorno de $4 \%$ 
Tabela 1. Volumes indicados para implantação de faixas adicionais (DMR, 2002)

\begin{tabular}{|c|c|c|c|c|}
\hline \multicolumn{2}{|c|}{$\begin{array}{c}\text { Oportunidades de ultrapassagens em } 5 \text { km que antecedem o trecho } \\
\text { em estudo }\end{array}$} & \multicolumn{3}{|c|}{ Volume Diário Médio (VDM) } \\
\hline \multirow{2}{*}{ Descrição } & \multirow{2}{*}{$\begin{array}{c}\text { Extensão com ultrapassagem permitida } \\
(\%)^{(\mathrm{b})}\end{array}$} & \multicolumn{3}{|c|}{ Porcentagem de veículos lentos ${ }^{(\mathrm{c})}$} \\
\hline & & $5 \%$ & $10 \%$ & $20 \%$ \\
\hline Excelente & $70-100$ & 4500 & 4000 & 3500 \\
\hline Bom & $30-70$ & 3500 & 3000 & 2600 \\
\hline Moderado & $10-30$ & 2500 & 2200 & 2000 \\
\hline Pouco moderado & $5-10$ & 1800 & 1600 & 1400 \\
\hline Restrito & $0-5$ & 1200 & 1000 & 900 \\
\hline Muito restrito $^{(\mathrm{d})}$ & 0 & 700 & 600 & 500 \\
\hline \multicolumn{5}{|c|}{$\begin{array}{l}\text { (a) A extensão do trecho antecedente pode variar entre } 3 \text { e } 10 \text { km (depende da rodovia em estudo); } \\
\text { (b) Consultar Chapter 9: - Road Planning and Design Manual (DMR, 2002); } \\
\text { (c) Inclui caminhões leves e automóveis com reboque; } \\
\text { (d) Sem oportunidades de ultrapassagens, nos dois sentidos, numa extensão de 3km. }\end{array}$} \\
\hline
\end{tabular}

sobre o custo de construção da faixa adicional, dentro do período de vida útil da obra.

Em Ontário, Canadá, nos aclives de rodovias em que o comprimento crítico de rampa for excedido, é preciso que a porcentagem de caminhões seja maior que $12 \%$ e o fluxo de veículos na hora de pico seja superior a 300 veíc/h, para que se justifique a implantação de uma faixa adicional (MTO, 1990).

No México, o Instituto Mexicano del Transporte (IMT) desenvolveu método de análise econômica para avaliar a implantação de faixas adicionais e estimar níveis de serviço, antes e após a construção dessas melhorias em rodovias de pista simples. A implantação da faixa adicional é economicamente viável se o volume diário médio estiver entre 3.000 veíc/dia e 6.000 veíc/dia e se a largura da plataforma da rodovia for suficiente para a construção da obra. Em caso contrário, os custos de terraplenagem podem inviabilizar a implantação da faixa adicional. Entretanto, para rodovias com volume diário médio superior a 6.000 veíc/dia, a faixa adicional deve ser implantada para aumentar a segurança durante as viagens (Mendonza e Mayoral, 1994).

\subsection{Critérios existentes no Brasil}

No Brasil, o Departamento Nacional de InfraEstrutura de Transportes (DNIT - antigo DNER) é o órgão responsável pela elaboração de diretrizes para projetos geométricos de rodovias rurais. O DNER dispõe de manual com diretrizes para implantação de terceira faixa, faixa de ultrapassagem, trecho de três faixas e baía de ultrapassagem como obras de melhorias para rodovias de pista simples (DNER, 1999). Os critérios para implantação de faixa adicional em aclives foram baseados nas diretrizes do Green Book da AASHTO (2001), sem esforços nem estudos para adaptá-los às condições das rodovias brasileiras.

\section{ADAPTAÇÃO DOS CRITÉRIOS DA AASHTO ÀS CONDIÇÕES BRASILEIRAS}

Com vistas a suprir a falta de diretrizes para implantação de faixas adicionais em rodovias no Brasil, Melo
(2002) fez adaptações aos critérios definidos pela AASHTO, às condições de tráfego e veículos brasileiros. Os aspectos adaptados foram: (i) curvas de desempenho para caminhões típicos da frota nacional; (ii) redução máxima admissível de velocidade em rampas para caminhões carregados; e (iii) fluxos mínimos de veículos e porcentagens de caminhões que justificam economicamente a construção da faixa adicional. Esses estudos podem ser vistos em Demarchi, Melo e Setti (2001) e em Melo e Setti (2002, 2003).

Para determinar o critério relativo aos fluxos mínimos, do qual trata este artigo, Melo (2002) estimou os benefícios gerados pela faixa adicional e os custos de construção para implantação da obra de melhoria.

Por análise econômica do investimento, através da relação benefício/custo, verificou-se que fluxos mínimos de veículos entre 180 e 340 veíc/h, justificam economicamente a construção da faixa adicional, em rampas com declividade entre $2,5 \%$ e $4 \%$ e porcentagens de caminhões entre $10 \%$ e $40 \%$. Mais detalhes pode ser vistos em Melo (2002), Melo e Setti (2003), e em Melo et al. (2006).

Entretanto, em função da falta de tempo e de uso de procedimentos manuais nas análises econômicas, no estudo de Melo (2002) não fora possível fazer uma análise paramétrica mais detalhada com vistas a propor uma maior gama de resultados. Desse modo, este artigo mostra os procedimentos efetuados para expandir os resultados obtidos por Melo (2002), com relação ao critério de fluxos mínimos de veículos, de maneira que o mesmo seja mais representativo das condições brasileiras e consista em aspecto importante para analisar implantação de faixas adicionais em aclives.

\section{CRITÉRIOS DE FLUXOS MÍNIMOS DE VEÍCULOS}

\subsection{Benefícios gerados com a implantação de faixa adicional}

Os benefícios gerados aos usuários com a implantação de faixas adicionais em aclives são redução dos tem- 
pos de viagem e dos custos de operação dos veículos, que podem ser medidos em valor monetário, e a redução da quantidade de acidentes, cuja valoração em termos monetários é mais complexa.

Na análise econômica foram incluídos os benefícios gerados pela redução do tempo de viagem e dos custos operacionais dos veículos. O ganho gerado pela redução dos acidentes não foi calculado, pois não existem estudos no Brasil que quantifiquem o benefício de redução de acidentes em função da construção da faixa adicional. Contudo, estudos mostram que a implantação de faixa adicional pode reduzir acidentes e os custos a eles relacionados (Stimpson e Glennon, 1971; Harwood et al., 1985; Jain e Taylor, 1991; AASHTO, 2001).

A estimativa dos benefícios foi feita a partir do ganho de velocidade média, da redução do tempo de viagem e da porcentagem de veículos em espera nos pelotões, quando implantada a faixa adicional. Essas informações foram obtidas através de simulações realizadas com o modelo de simulação para tráfego de veículos em vias de pistas simples, o TRARR. O motivo da escolha do simulador TRARR como ferramenta para obtenção e cálculo dos benefícios foi a disponibilidade de versão calibrada por Egami (2000), de acordo com as condições típicas das rodovias do estado de São Paulo.

O simulador TRARR é um modelo de simulação microscópico e estocástico capaz de simular um fluxo de tráfego ininterrupto em uma rodovia de pista simples, "sem" ou "com" faixas adicionais e sem interseções. O modelo foi desenvolvido, entre anos de 1978 e 1980, por Geoff Robinson do AARB Transport Research Ltd. da Austrália (Hoban et al., 1991; Egami, 2000). No modelo podem ser avaliadas as alterações causadas à operação da rodovia, seja por modificações efetuadas na geometria ou nos parâmetros relacionados ao tráfego de veículos.

\subsection{Custos com a implantação de faixas adicionais}

As alternativas de construção de uma faixa adicional variam de um simples reforço do acostamento existente até soluções mais completas, como a construção de uma faixa extra com acostamento. Os custos de construção irão depender da alternativa escolhida, pois implicam na inclusão ou exclusão de itens que compõem a obra. Segundo diversos autores (DNER, 1979; GEIPOT, 1982; Kabbach, 1992; Pedrozo et al., 2001), os custos de construção em obras rodoviárias dependem de fatores relacionados ao:

- Terreno: características topográficas e geotécnicas do local;

- Projeto: disponibilidade de materiais e respectivas distâncias de transportes, seção transversal adotada, volumes de corte e de aterro, reforço ou demolição da estrutura do acostamento, drenagem, sinalização, defensas e desapropriações;

- Administração da obra: provisões para contingências e custos de projeto, e supervisão da obra.

Contudo, como a adoção de faixa adicional é uma obra de melhoria de baixo custo, esse tipo de solução é indicado para aclives onde os custos sejam os mínimos possíveis, tais como, locais em que a plataforma esteja pronta, o volume de terra movimentado seja pequeno ou com material de fácil escavação e remoção (material de $1^{\underline{a} \text {. }}$ categoria).

O custo de manutenção causado pela implantação da faixa adicional consiste em uma série de atividades preventivas e corretivas - tais como, operação tapaburacos, reacapeamento, manutenção da sinalização, da drenagem entre outros - que visam minimizar a deterioração e manter a obra em condições de trafegabilidade e segurança. Os custos de operação, como de centrais de controle de tráfego e terminais, podem ser desprezados, pois são custos considerados apenas em sistemas de transportes urbanos (DNER, 1979; Kabbach, 1992).

\subsection{Caracterização dos trechos simulados}

Para cumprir o objetivo proposto, diversos cenários foram elaborados com o intuito de se efetuar combinações entre parâmetros de tráfego (fluxos de veículos e porcentagens de caminhões), geometria da rodovia (declividade e comprimento do aclive) e implantação da obra de melhoria ("não fazer nada” ou construção da faixa adicional).

Com relação ao tráfego, os fluxos de veículos em horário de pico foram analisados entre 50 veíc/h e 900 veíc/h, com distribuição por sentido admitida como sendo $60 \% / 40 \%$, nos sentidos ascendente e descendente, respectivamente.

Foram considerados apenas duas configurações de caminhões (rígido pesado e articulado pesado), visto que ambos representam cerca de $80 \%$ dos caminhões que trafegam em rodovias do estado de São Paulo (Melo, 2002). Dentro da composição do tráfego, a parcela de caminhões foi analisada entre $10 \%$ e $40 \%$, dos quais foram admitidos que 50\% estariam dentro do limite de peso bruto total permitido por lei e os demais com excesso de carga. Os valores adotados para a relação massa/potência podem ser vistos na Tabela 2.

O caminhão rígido pesado, com sobrecarga e relação massa/potência de $300 \mathrm{~kg} / \mathrm{kW}$, foi escolhido como o veículo de projeto, pois é o que apresenta pior desempenho dentre os caminhões analisados.

Os segmentos de rodovias simulados compreenderam trechos em tangente compostos de dois subtrechos planos (comprimento de $1.000 \mathrm{~m}$ e $3.000 \mathrm{~m}$ ) e 
Tabela 2. Relação massa/potência de caminhões brasileiros (kg/kW)

\begin{tabular}{llll}
\hline Configuração & Denominação & Limite legal & Com sobrecarga \\
\hline & Rígido pesado & 230 & 300 \\
\hline
\end{tabular}

um aclive (comprimento e declividade variáveis). A Figura 1 mostra a geometria dos trechos de rodovia simulados.

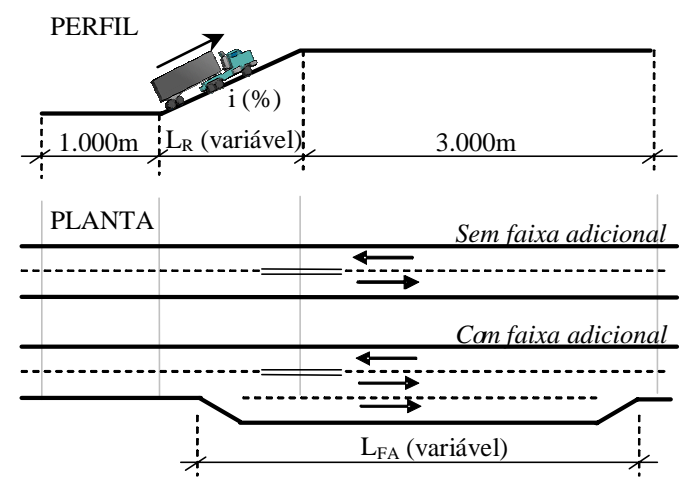

Figura 1. Geometria dos trechos simulados (sem escala)

Os elementos que caracterizam a faixa adicional foram definidos conforme se segue. O ponto de início corresponde ao local em que o caminhão de projeto (rígido pesado sobrecarregado) atinge $70 \mathrm{~km} / \mathrm{h}$, ou seja, diferença entre a velocidade máxima permitida no trecho antecedente $(90 \mathrm{~km} / \mathrm{h})$ e a máxima redução admissível de velocidade (20 km/h). Da mesma forma, o ponto final é o local em que o caminhão retoma a velocidade de entrada na faixa adicional. A extensão percorrida pelo caminhão com velocidades inferiores a $70 \mathrm{~km} / \mathrm{h}$ define o comprimento da faixa adicional, que variaram de $2.900 \mathrm{~m}$ a $5.000 \mathrm{~m}$. Embora o comprimento da faixa adicional seja grande, se comparado aos $1.200 \mathrm{~m}$ ou $3.000 \mathrm{~m}$ propostos em outros estudos (DMR, 2002; ATU, 1995; respectivamente), deve-se notar que neste estudo foi considerado um veículo de projeto com excesso de carga (300 kg/kW), um veículo de baixo desempenho que necessita de grandes distâncias para retomada de velocidade, bem diferente do caminhão de projeto com relação massa/potência de $180 \mathrm{~kg} / \mathrm{kW}$ daqueles estudos.

Como foi adotado um procedimento estatístico para análise de valores médios dos parâmetros usados no cálculo dos benefícios, cada combinação entre fluxo de veículos, composição de tráfego, declividade e comprimento de rampa foi simulada cinco vezes. A análise paramétrica efetuada totalizou em mais de 20.000 simulações, conforme a Tabela 3.

O TRARR gera arquivos de saída denominados out(\#), em que \# é o identificador da quantidade de arquivos simulados. Como os arquivos de saída gerados não possuem extensão compatível com Windows, não é possível abrí-los diretamente de um programa como o Excel para realização das análises e é difícil relacionar o arquivo de saída com o cenário simulado. Desse modo, os arquivos de saída eram renomeados com extensão compatível ao WordPad; após renomeados, eram selecionados e abertos, dos quais eram copiados dados relativos ao sentido ascendente da rodovia em análise: fluxos de veículos, tempo de viagem, velocidade média e porcentagem de veículos em pelotões. A seguir, as informações copiadas eram coladas em grupos de cinco numa planilha Excel, na qual era efetuado o cálculo dos valores médios de simulação. Na última etapa do processamento, uma segunda planilha era usada para calcular os benefícios obtidos pela redução dos custos operacionais dos veículos e dos tempos de viagem e realizar a análise econômica da construção da faixa adicional. Para isso, os valores médios obtidos na terceira etapa eram copiados nesta segunda planilha eletrônica.

Com todos esses procedimentos manuais para recuperação dos arquivos de saída e análises de resultados do TRARR, o tempo gasto no processamento é relativamente longo. Desse modo, para aperfeiçoar o processamento, foi elaborado um software denominado SunSet com a finalidade de reduzir o tempo de análise dos resultados obtidos nas rodadas de simulações e proporcionar análises econômicas mais abrangentes (Melo e Justino, 2005; Melo et al., 2006).

\subsection{Análise econômica}

Os benefícios gerados com a implantação da faixa adicional (redução do tempo de viagem e de custos operacionais) foram estimados por procedimentos descritos na literatura (DNER, 1976, 1979; EBTU, 1981; Kabbach, 1992; Melo, 2002; Economia \& Transporte, 2006). O cálculo dos benefícios foi feito

Tabela 3. Quantidade de cenários simulados

\begin{tabular}{ll}
\hline Parâmetro & Quantidade \\
\hline Fluxos (veíc/h): 50, 100, 150, 200, 250, 300, 350, 400, 500, 600, 700, 800, 900 & 13 \\
Composição de caminhões no tráfego (\%): 10, 20, 25, 30, 35, 40 & 6 \\
Declividade da rampa (\%): 2,5; 3, 4, 5, 6, 7, 8 & 7 \\
Comprimento da rampa (m): 500, 1.000, 1.500, 2.000 & 4 \\
Faixa adicional (cenário 0: sem faixa; cenário 1: com faixa) & 2 \\
Número de replicações por arquivo & 5 \\
\hline Número total de cenários simulados $=13 \times 6 \times 7 \times 4 \times 2 \times 5=$ & 21.840 \\
\hline
\end{tabular}


com resultados sobre ganho de velocidade média, redução da porcentagem de veículos em pelotões e dos tempos de viagem obtidos nas simulações.

Os benefícios gerados pela redução de acidentes não foram considerados, pois não existem estudos no Brasil que retratem a redução no número de acidentes em função da construção da faixa adicional.

Da soma entre os benefícios de redução de custos operacionais dos veículos e de redução dos tempos de viagens foram estimados os benefícios anuais totais:

$$
B T=B R C O+B R T V
$$

em que $\quad B T$ : Benefício anual total ( $\mathrm{R} \$ / \mathrm{ano})$;

BRCO: Benefício de redução dos custos operacionais dos veículos ( $\mathrm{R} \$ / \mathrm{ano})$;

BRTV: Benefício de redução dos tempos de viagem ( $\mathrm{R} \$$ /ano).

Os custos associados à implantação da faixa adicional foram considerados como a soma do custo de construção e custo de manutenção, estimado em $10 \%$ do custo de construção para vida útil da faixa adicional (10 anos). Os custos de construção foram estimados a partir de quantidades de materiais e serviços obtidos em uma concessionária de rodovias do estado de São Paulo e de custos unitários da Tabela de Preços Unitários do DER-SP, edição de maio 2005 (DER, 2005). Admitiu-se que a plataforma para a execução da faixa adicional estava pronta para ser pavimentada e, por conseguinte, a parcela de custo de terraplenagem foi desprezada. Essa simplificação foi adotada por duas razões: (i) é difícil determinar um volume de terraplenagem; (ii) faixas adicionais são obras de melhoria viária de baixo custo e, por isso, devem ser construídas em locais onde a movimentação de terra é a mínima possível. Caso as condições do local exijam a construção de obras de arte ou execução de terraplenagem, os fluxos mínimos e porcentagens de caminhões determinados nesta análise não serão suficientes para avaliar o retorno econômico e uma análise mais detalhada deverá ser feita.

A análise econômica do investimento foi feita pela relação benefício/custo, por se tratar de um método bastante usado e de fácil uso, além de facilitar comparações entre diferentes alternativas de projeto. A relação benefício/custo (B/C) consiste em estimar a razão entre os benefícios e os custos totais. Um projeto é considerado economicamente viável se a relação B/C for maior ou igual a unidade e, quanto maior a relação, mais atraente é o projeto. Assim, o fluxo mínimo que justifica economicamente a implantação da faixa adicional é aquele para o qual a relação B/C é igual a um, garantindo a viabilidade econômica da faixa adicional. Os resultados obtidos estão resumidos na Tabela 4 .

A Figura 2 mostra a variação dos fluxos mínimos de veículos, em função do comprimento, declividade da rampa e porcentagens de caminhões carregados na composição do tráfego.

Pelos resultados apresentados, pode-se constatar que os fluxos mínimos de veículos, para justificar a

Tabela 4. Fluxos mínimos, na hora de pico e no sentido ascendente, que justificam a implantação de faixa adicional em aclives (veic/h)

\begin{tabular}{|c|c|c|c|c|c|c|c|}
\hline \multirow[b]{2}{*}{ Rampa (\%) } & \multirow[b]{2}{*}{ Comprimento (m) } & \multicolumn{6}{|c|}{ Porcentagem de caminhões (\%) } \\
\hline & & 10 & 20 & 25 & 30 & 35 & 40 \\
\hline \multirow{4}{*}{2,5} & 500 & 540 & 520 & 480 & 420 & 440 & 450 \\
\hline & 1.000 & 530 & 500 & 460 & 400 & 420 & 435 \\
\hline & 1.500 & 510 & 480 & 440 & 385 & 400 & 415 \\
\hline & 2.000 & 490 & 460 & 420 & 370 & 390 & 405 \\
\hline \multirow{4}{*}{3} & 500 & 490 & 470 & 430 & 390 & 410 & 420 \\
\hline & 1.000 & 480 & 450 & 410 & 375 & 395 & 405 \\
\hline & 1.500 & 470 & 430 & 390 & 350 & 370 & 390 \\
\hline & 2.000 & 450 & 410 & 370 & 340 & 360 & 375 \\
\hline \multirow{4}{*}{4} & 500 & 460 & 440 & 420 & 380 & 390 & 395 \\
\hline & 1.000 & 440 & 420 & 400 & 365 & 375 & 380 \\
\hline & 1.500 & 435 & 400 & 380 & 345 & 350 & 365 \\
\hline & 2.000 & 400 & 380 & 360 & 330 & 340 & 350 \\
\hline \multirow{4}{*}{5} & 500 & 420 & 410 & 400 & 360 & 370 & 370 \\
\hline & 1.000 & 400 & 375 & 350 & 340 & 345 & 350 \\
\hline & 1.500 & 400 & 380 & 360 & 340 & 345 & 350 \\
\hline & 2.000 & 360 & 340 & 320 & 300 & 305 & 310 \\
\hline \multirow{4}{*}{6} & 500 & 400 & 370 & 360 & 350 & 360 & 370 \\
\hline & 1.000 & 370 & 340 & 340 & 320 & 330 & 350 \\
\hline & 1.500 & 360 & 340 & 320 & 305 & 315 & 340 \\
\hline & 2.000 & 330 & 310 & 300 & 290 & 300 & 330 \\
\hline \multirow{4}{*}{7} & 500 & 370 & 340 & 320 & 315 & 315 & 320 \\
\hline & 1.000 & 340 & 300 & 290 & 280 & 290 & 300 \\
\hline & 1.500 & 330 & 300 & 290 & 270 & 280 & 290 \\
\hline & 2.000 & 300 & 275 & 265 & 260 & 275 & 280 \\
\hline \multirow{4}{*}{8} & 500 & 310 & 290 & 270 & 250 & 255 & 260 \\
\hline & 1.000 & 300 & 280 & 260 & 250 & 255 & 260 \\
\hline & 1.500 & 300 & 280 & 260 & 240 & 255 & 270 \\
\hline & 2.000 & 280 & 265 & 250 & 230 & 235 & 245 \\
\hline
\end{tabular}



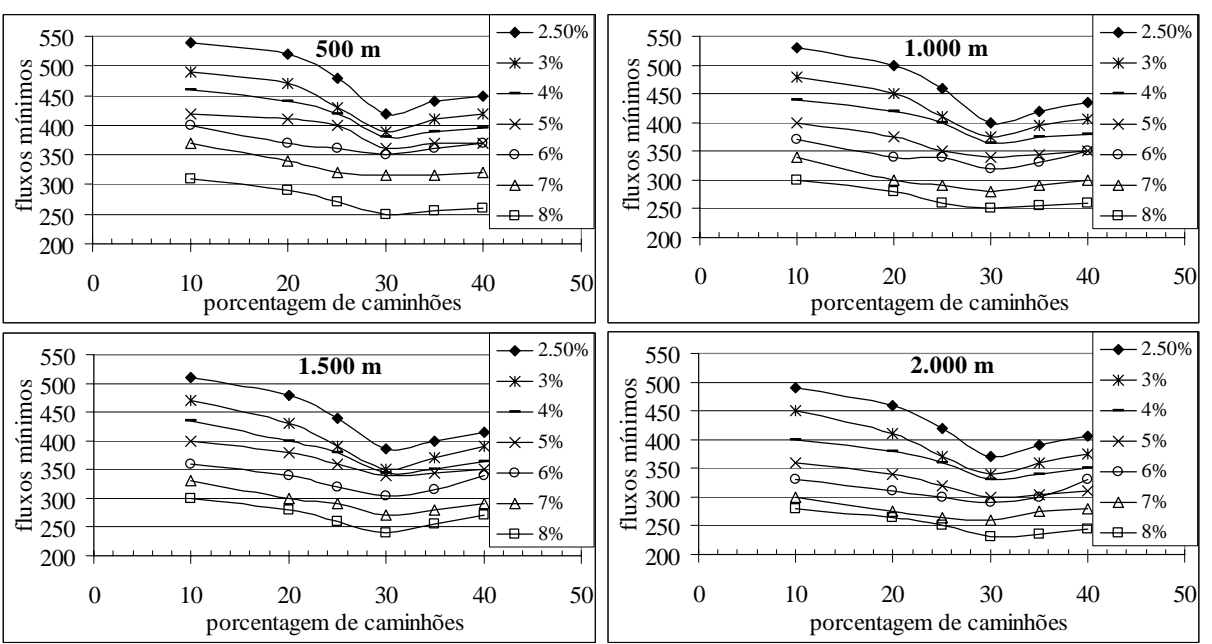

Figura 2. Fluxos mínimos de veículos (hora de pico e sentido ascendente) em função geometria do aclive e porcentagens de caminhões

construção da faixa adicional, dependem da geometria da rampa ascendente e das porcentagens de caminhões que compõem o tráfego. Levando-se em conta as características geométricas, os fluxos de veículos diminuem à medida que a declividade e o comprimento da rampa aumentam. Com relação ao tráfego, os fluxos de veículos são decrescentes até que os caminhões atinjam 30\% dentro da composição do tráfego, a partir daí, os fluxos de veículos aumentam, pois os benefícios começam a se reduzir já que a quantidade de veículos rápidos (carros e ônibus) vai se tornando muito pequena para garantir o retorno do investimento.

\section{CONSIDERAÇÕES FINAIS}

O estudo realizado produziu um critério de fluxos mínimos de veículos para verificação da necessidade de faixas adicionais em aclives de rodovias de pista simples, expandindo uma análise preliminar feita por Melo (2002). Com os resultados obtidos, foi possível adaptar mais um critério das diretrizes da AASHTO (2001) às condições de tráfego e veículos brasileiros. O critério de fluxos mínimos de veículos juntamente com os critérios de máxima redução admissível da velocidade do caminhão $(20 \mathrm{~km} / \mathrm{h})$ e curvas de desempenho para caminhões típicos do Brasil constitui um conjunto de diretrizes que podem permitir a verificação da necessidade de investimentos em obras de melhoria de baixo custo, com vistas a melhorar o nível de serviço e reduzir índices de acidentes em rodovias brasileiras.

Entretanto, o uso das diretrizes deve ser acompanhado de análises um pouco mais amplas, visto que o TRARR foi calibrado com características de veículos e de tráfego existentes em rodovias do estado de São Paulo, o que pode não representar as condições existentes em rodovias de outros estados brasileiros.

\section{REFERÊNCIAS BIBLIOGRÁFICAS}

AASHTO (2001) Elements of Design. In: A Policy on Geometric Design of Highways and Streets. American Association of State Highway and Transportation Officials. p. 235-254. Fourth Edition. Washington, D. C..

ATU (1995) Alignment Elements. In: Highway Geometric Design Guide. Alberta Infrastructure, Alberta Transportation and Utilities. p. B55 to B-79. Alberta, Canadá.

CNT (2006) Pesquisa Rodoviária 2006: Relatório Gerencial. Confederação Nacional do Transporte. Disponível em : <http://www.cnt. org.br>. Acessado em 15 de março de 2002.

Demarchi, S. H.; R. A. Melo e J. R. A. Setti (2001) Validação de Um Modelo de Desempenho de Caminhões em Rampas Ascendentes. Revista Transportes, vol. IX, n. 1, p. 51-68.

DENATRAN (2007). Registro Nacional de Acidentes e Estatísticas de Trânsito - RENAEST. DENATRAN - DEPARTAMENTO NACIONAL DE TRÂNSITO. Disponível em: <http:// www.infoseg.gov.br/renaest>. Acessado em 26 de junho de 2007.

DER (2005) Tabela de Preços Unitários. Data de referência 31/05/2005. Departamento de Estradas de Rodagem do estado de São Paulo. São Paulo, SP. Disponível em: <http://www.der.sp.gov.br/obras/ frm_obr.htm>. Acessado em 6 de março de 2006.

DMR (2002) Auxiliary Lanes. In: Road Planning and Design Manual. Queensland Department of Main Roads. Queensland Government. Queensland, Austrália.

DNER (1976) Manual de Custo de Operação. Departamento Nacional de Estradas de Rodagem. Rio de Janeiro, Brasil.

DNER (1979) Instruções para Implantação de Terceiras Faixas. Departamento Nacional de Estradas de Rodagem. Rio de Janeiro.

DNER (1999) Terceira Faixa nas Rampas Ascendentes. In: Manual de Projeto Geométrico de Rodovias Rurais. pg. 177-186. Departamento Nacional de Estradas de Rodagem. Rio de Janeiro.

EBTU (1981) Manual de Avaliação de Projetos. Empresa Brasileira de Transportes Urbanos (EBTU). Brasília.

Economia \& Transporte (2006) Custos Operacionais. Disponível em: $<$ http://www.economiaetransporte.com.br/index2.html>. Acessado em 6 de março de 2006.

Egami, C. Y. (2000) Recalibração de um Modelo para Simulação do Tráfego em Rodovias de Pista Simples. 137 p. Dissertação (Mestrado) - Escola de Engenharia de São Carlos, Universidade de São Paulo. S. Carlos, S. Paulo.

Ferreira, C. E. C. (2003) Acidentes com Motoristas no Transporte Rodoviário de Produtos Perigosos. Revista São Paulo em Perspectiva, v. 17, n. 2, p. 68-80.

GEIPOT (1982) Pesquisa sobre o Inter-relacionamento dos Custos de Construção, Conservação e Utilização de Rodovias: Relatório Final. Empresa Brasileira de Planejamento de Transportes. Rio de Janeiro.

Harwood, D. W., A. D. St. John e D. L. Warren (1985) Operational and Safety Effectiveness of Passing Lanes on Two-Lane Highways. Transportation Research Record, v. 1026, p. 31-39. Washington, D. C.. 
Hoban, C. J. et al. (1991) A Model for Simulating Traffic on Two-Lane Roads - User Guide and Manual for TRARR Version 3.2. ARRB Transport Research Ltd.. Victoria - Australia.

Jain, M. K. e W. C. Taylor (1991) Criteria for passing relief lanes on twolane highways. ITE Journal, v. 61, n. 2, p. 25-30, feb. Institute of Transportation Engineers. Washington, D.C.

Kabbach, F. I. J. (1992) Contribuição para o Estudo de Implantação de Faixas Adicionais em Rampas Ascendentes de Rodovias de Pista Simples. 333 p. Tese (Doutorado) - Escola Politécnica, Universidade de São Paulo. S. Paulo.

Melo, R. A. (2002) Faixas Adicionais para Aclives de Rodovias Brasileiras. 175 p. Tese (Doutorado) - Escola de Engenharia de São Carlos, Universidade de São Paulo. São Carlos, São Paulo.

Melo, R. A. e J. Justino (2005) SunSet: Programa de Recuperação e Análise dos Dados de Saída do Simulador de Tráfego TRARR - versão 1.0.4. 21 p.. Manual do Usuário - Universidade Federal da Paraíba, Departamento de Engenharia Civil. João Pessoa.

Melo, R. A.; J. Justino e J. R. A. Setti (2006) Implantação De Faixas Adicionais Em Aclives De Rodovias: Otimização Do Critério De Fluxos Mínimos De Veículos. Anais do XX Congresso de Pesquisa e Ensino em Transportes, ANPET, Brasília, Cd-Rom, v. 1, comunicação técnica, 8 p..

Melo, R. A. e J. R. A. Setti (2002) Critérios para Implantação de Faixas Adicionais em Rampas Ascendentes de Rodovias Brasileiras. Anais do XII Congreso Panamericano de Ingeniería de Trânsito y Transporte, PANAM, Quito, Cd-Rom, 13 p..

Melo, R. A. e J. R. A. Setti (2003) Faixas Adicionais para Aclives de Rodovias de Pista Simples Brasileiras: Uma Adaptação dos Critérios da AASHTO. Revista Transportes, vol. XI, n. 2, p. 41-48.

Mendonza, A. e E. Mayoral (1994) Economic Feasibility Assessment Procedure for Climbing Lanes on Two-Lanes Roads in Mexico. Transportation Research Record, v. 1457, p. 26-34. Washington, D. C..

MTO (1990) Heavy Vehicle Performance on Grade and Climbing Lane Criteria. Documentation Page TDS-90-11. Ministry of Transportation. Ontário.

Pedrozo, L. G; L. A. S. Senna e F. D. Michel (2001) Custos Rodoviários Análise e Sistematização. Departamento Autônomo de Estradas de Rodagem do estado do Rio Grande do Sul (DAER). Revista Estradas, n. 1, p. 16-23.

Stimpson, W. A. e J. C. Glennon (1971) Critical Review of Climbing Lane Design Practices. Highway Research Board Bulletin 167, p. 1-11, Washington, D.C. 